\title{
Effects of vitamin C supplementation on the blood oxidative stress and antibody titre against Histophilus somnivaccination in calves
}

\author{
Konosuke Otomaru ${ }^{\bowtie}$, Shoko Oishi, Yu Fujimura, \\ Yuki Iwamoto, Katsuhisa Nagai, Moe Ijiri \\ Joint Faculty of Veterinary Medicine, Kagoshima University, Kagoshima 890-0065, Japan \\ otomaru@vet.kagoshima-u.ac.jp
}

Received: July 6, $2020 \quad$ Accepted: December 7, 2020

\begin{abstract}
Introduction: The purpose of this study was to determine the effects of vitamin C supplementation on blood oxidative stress biomarkers and antibody response to vaccination in calves. Material and methods: Thirty-four clinically healthy 2 week old Japanese Black calves were randomly assigned to two groups. Seventeen calves formed the VC group which received 1,000 mg of vitamin $\mathrm{C}$ daily from 2 to 8 weeks of age, and the other 17 calves of the control group did not receive supplementation. All calves received an inactivated Histophilus somni vaccine at 4 and 8 weeks of age. Blood samples were taken at 2, 4, 8 and 12 weeks of age. Results: The concentration of the serum reactive oxygen metabolites (d-ROMs), and the oxidative stress index (OSI), which is calculated from the d-ROMs and biological antioxidant potential, were significantly lower at 8 weeks of age in the VC group than in the control group $(\mathrm{P}<0.05)$. The antibody titres to $H$. somni in the VC group were significantly higher than those in the control group at 12 weeks of age after the second vaccination $(\mathrm{P}<0.05)$. Conclusion: Vitamin $\mathrm{C}$ supplementation to calves may reduce oxidative stress and enhance the antibody production after vaccination with $H$. somni.
\end{abstract}

Keywords: calves, Histophilus somni, oxidative stress, vaccination, vitamin C.

\section{Introduction}

Young suckling calves have immature immune systems as evidenced by their lower numbers of lymphocytes in the peripheral blood, which are responsible for humoral and cellular immunity (9). This was demonstrated by a weak antibody response to vaccination (16). Additionally, calves of Japanese Black cattle have a lower number of immune cells than other breeds and thus tend to be more susceptible to diseases compared to other cattle breeds (17).

Histophilus somni is a common inhabitant bacterium of the upper respiratory tract of healthy cattle (2), and has been most commonly isolated in bovine respiratory diseases $(2,6)$. Antimicrobial agents are generally used to treat bacterial infections. However, in recent years, their use in livestock animals has been restricted. To prevent respiratory infections in cattle by bacteria such as $H$. somni, vaccination has precedence over antibiotic therapy. Thus, in Japan, almost all Japanese Black calves are vaccinated with an $H$. somni preparation.
Vitamin $\mathrm{C}$ is an important free radical scavenger and as an antioxidant, acts inside the body to stabilise biological membranes and maintain immune function (4, 15, 22). Many studies investigating vitamin C administration have reported more efficient quenching of singlet oxygen and strengthened immunity $(4,15,20)$. Therefore, supplementation of vitamin $\mathrm{C}$ to Japanese Black calves was expected to reduce oxidative stress and improve immune function. The purpose of this study was to evaluate the beneficial effects of such supplementation on the serum oxidative stress biomarkers and the antibody response to inactivated H. somni vaccination in Japanese Black calves.

\section{Material and Methods}

Thirty-four clinically healthy 2-week-old Japanese Black calves kept on one farm in Kagoshima Prefecture, Japan, were used in this study. All the calves stayed with their mothers for 4 days after birth and were housed indoors. Starting at 5 days after birth, they were fed with 
milk replacer and were raised individually in calf hutches. The amount and nutrient composition of feed are shown in Table 1. The husbandry of all calves was unvaried, they were weaned at 12 weeks of age and fed to meet their nutritional requirements according to the Japanese beef cattle feeding standard (13). The calves were randomly assigned to two groups. Seventeen calves were orally supplemented with a dose based on the study by Hemingway (7) of $1000 \mathrm{mg}$ of vitamin C (Rovimix vitamin C; DSM Nutrition Products, Basel, Switzerland) once daily from 2 to 8 weeks of age (VC group), and 17 calves were not supplemented with vitamin $\mathrm{C}$ (control group). All calves were vaccinated with a commercially available inactivated $H$. somni vaccine (M-1 Br strain; Kyoto Biken Laboratories Inc., Kyoto, Japan) at 4 and 8 weeks of age following the manufacturer's instructions. All the calves ate all the provided feed, and no calves developed disease during the experiment. Blood samples were taken at 2, 4, 8 and 12 weeks of age from the jugular vein using Vacutainer tubes containing dipotassium edetic acid (EDTA-2AK) and plain Vacutainer tubes. Serum was isolated from the blood samples in plain tubes by centrifugation and stored at $-30^{\circ} \mathrm{C}$ until analysis.

White blood cells (WBC) and red blood cells (RBC) were counted and haemoglobin ( $\mathrm{Hb})$ and haematocrit $(\mathrm{Ht})$ were measured in the blood samples in the EDTA-2AK tubes using a 6550 automatic haemocytometer (Nihon Kohden, Tokyo, Japan) within $4 \mathrm{~h}$ of collection. The serum oxidant status was determined using a diacron reactive oxygen metabolites (d-ROMs) test, which determines hydroperoxides $(1,18$, 21). The serum concentrations of antioxidant capacity were measured using a biological antioxidant potential (BAP) test $(1,18,21)$, and the d-ROMs and BAP were measured using a free radical analyser (FREE Carrio Duo, Diacron International, Grosseto, Italy) $(1,18)$. The degree of oxidative stress was expressed as the oxidative stress index (OSI) calculated with the formula $(\mathrm{d}-\mathrm{ROMs} / \mathrm{BAP}) \times 100=$ OSI $(1,21)$. Serum antibody titres against $H$. somni were detected in ELISA, as described in a previous study $(3,14)$. H. somni of the M-1 Br strain was dispensed into wells of a microtitre plate, and serum samples at 400 -fold dilution were added to the wells and incubated, at $30^{\circ} \mathrm{C}$ for $1 \mathrm{~h}$. After washing, peroxidase-conjugated anti-bovine $\operatorname{IgG}$ was added and the samples were incubated again, at $30^{\circ} \mathrm{C}$ for $30 \mathrm{~min}$. After a second washing, o-phenylenediamine was added in a citrate-phosphate buffer and the plates were incubated once again, at $30^{\circ} \mathrm{C}$ for $30 \mathrm{~min}$. The reaction was stopped and the optical density was read at $492 \mathrm{~nm}$, using reference wavelength of $630 \mathrm{~nm}$.

Data were expressed as the mean \pm standard error. Statistical analysis was conducted to determine the differences between the two groups in the same weeks of age using Student's $t$-test with SPSS statistics 24 software (IBM, Tokyo, Japan). P values less than 0.05 were considered statistically significant.

\section{Results}

The values of WBC, RBC, $\mathrm{Hb}$ and $\mathrm{Ht}$ were not significantly different between the groups (Table 2). The serum d-ROMs concentration in the control group was higher than that in the VC group from 2 to 12 weeks of age (Fig. 1), and the difference between the groups was statistically significant at 8 weeks $(\mathrm{P}<0.05)$. The serum BAP was not significantly different between the groups (Fig. 2). However, the OSI in the control group was significantly higher than that in the VC group at 8 weeks of age ( $\mathrm{P}<0.05)$ (Fig. 3). Fig. 4 shows the changes in the serum antibody titres against $H$. somni. In the VC group, the antibody titres against $H$. somni were significantly higher than those in the control group at 12 weeks of age after the second vaccination $(\mathrm{P}<0.05)$.

Table 1. Amount and nutrient composition of feed without supplement (per head per day)

\begin{tabular}{|c|c|c|c|c|}
\hline & \multicolumn{4}{|c|}{ Weeks of age } \\
\hline & 2 & 4 & 8 & 12 \\
\hline \multicolumn{5}{|l|}{ Amount (dry matter; kg) } \\
\hline Milk replacer & 0.90 & 1.08 & 1.08 & 0.00 \\
\hline Concentrate & 0.04 & 0.09 & 0.45 & 1.08 \\
\hline Oats & 0.01 & 0.01 & 0.08 & 0.16 \\
\hline \multicolumn{5}{|l|}{ Composition (dry basis; \%) } \\
\hline Total digestible nutrients & 103.4 & 102.4 & 95.8 & 77.5 \\
\hline Crude protein & 27.4 & 27.1 & 24.9 & 18.8 \\
\hline Crude fat & 15.5 & 15.1 & 11.8 & 2.2 \\
\hline Calcium & 1.34 & 1.31 & 1.13 & 0.61 \\
\hline Phosphorous & 0.72 & 0.71 & 0.66 & 0.52 \\
\hline Magnesium & 0.11 & 0.11 & 0.14 & 0.21 \\
\hline
\end{tabular}


Table 2. Haematological parameters

\begin{tabular}{|c|c|c|c|c|c|}
\hline \multirow{2}{*}{ Parameter } & & \multicolumn{4}{|c|}{ Weeks of age } \\
\hline & & 2 & 4 & 8 & 12 \\
\hline \multirow{2}{*}{ White blood cells $\left(10^{2}\right.$ cell $\left./ \mu \mathrm{L}\right)$} & VC group & $86.0 \pm 5.4$ & $80.4 \pm 3.6$ & $81.6 \pm 5.4$ & $82.4 \pm 5.9$ \\
\hline & Control group & $79.6 \pm 5.3$ & $88.4 \pm 4.1$ & $88.2 \pm 5.5$ & $83.8 \pm 3.2$ \\
\hline \multirow{2}{*}{ Red blood cells $\left(10^{4}\right.$ cell $\left./ \mu \mathrm{L}\right)$} & VC group & $846 \pm 23$ & $948 \pm 30$ & $1022 \pm 22$ & $1057 \pm 20$ \\
\hline & Control group & $893 \pm 29$ & $1007 \pm 26$ & $1102 \pm 24$ & $1099 \pm 36$ \\
\hline \multirow{2}{*}{ Haemoglobin (g/dL) } & VC group & $9.8 \pm 0.2$ & $10.5 \pm 0.4$ & $10.9 \pm 0.2$ & $11.2 \pm 0.2$ \\
\hline & Control group & $9.9 \pm 0.3$ & $10.6 \pm 0.3$ & $11.4 \pm 0.2$ & $11.7 \pm 0.2$ \\
\hline \multirow{2}{*}{ Haematocrit (\%) } & VC group & $31.9 \pm 0.8$ & $33.6 \pm 1.1$ & $34.4 \pm 0.7$ & $35.3 \pm 0.7$ \\
\hline & Control group & $32.1 \pm 1.0$ & $33.6 \pm 0.9$ & $35.6 \pm 0.7$ & $35.3 \pm 0.8$ \\
\hline
\end{tabular}

Data are shown as mean $\pm \mathrm{SE}$

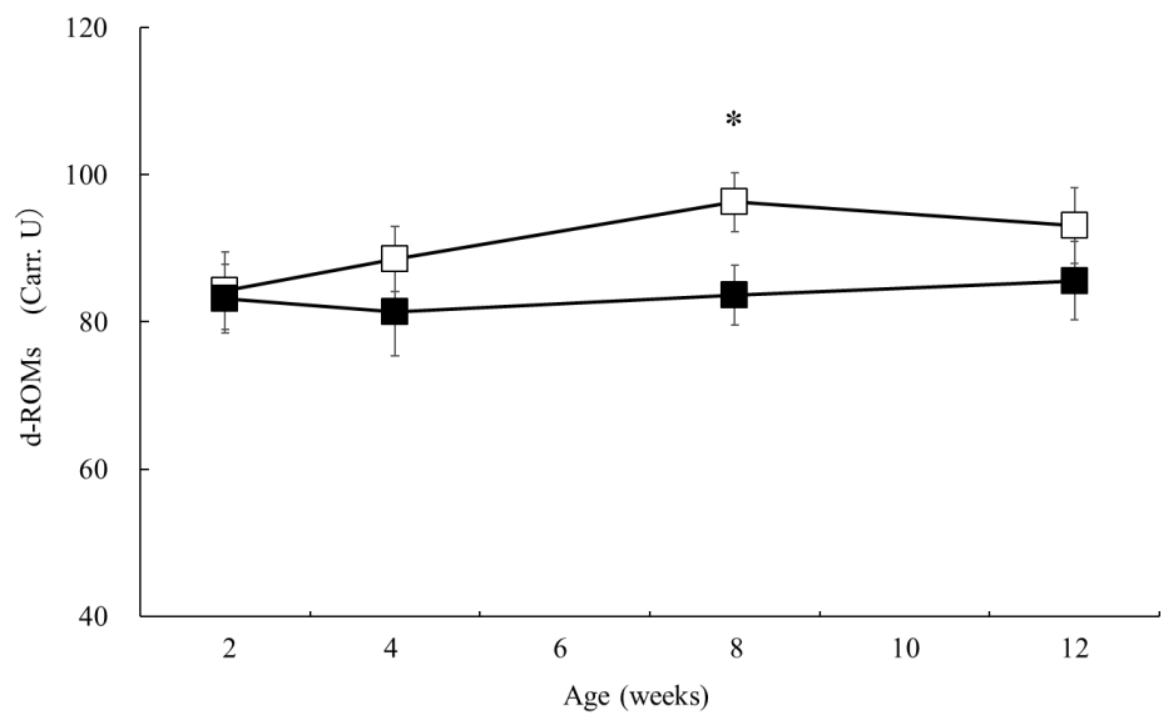

Fig. 1. Changes in serum derivatives of reactive oxygen metabolites (d-ROMs) in the VC group (solid squares) and the control group (hollow squares)

The amount of d-ROMs corresponds to the amount of hydrogen peroxide

The asterisk indicates a significant difference between groups at the same age $(\mathrm{P}<0.05)$

Data are shown as mean $\pm \mathrm{SE}$

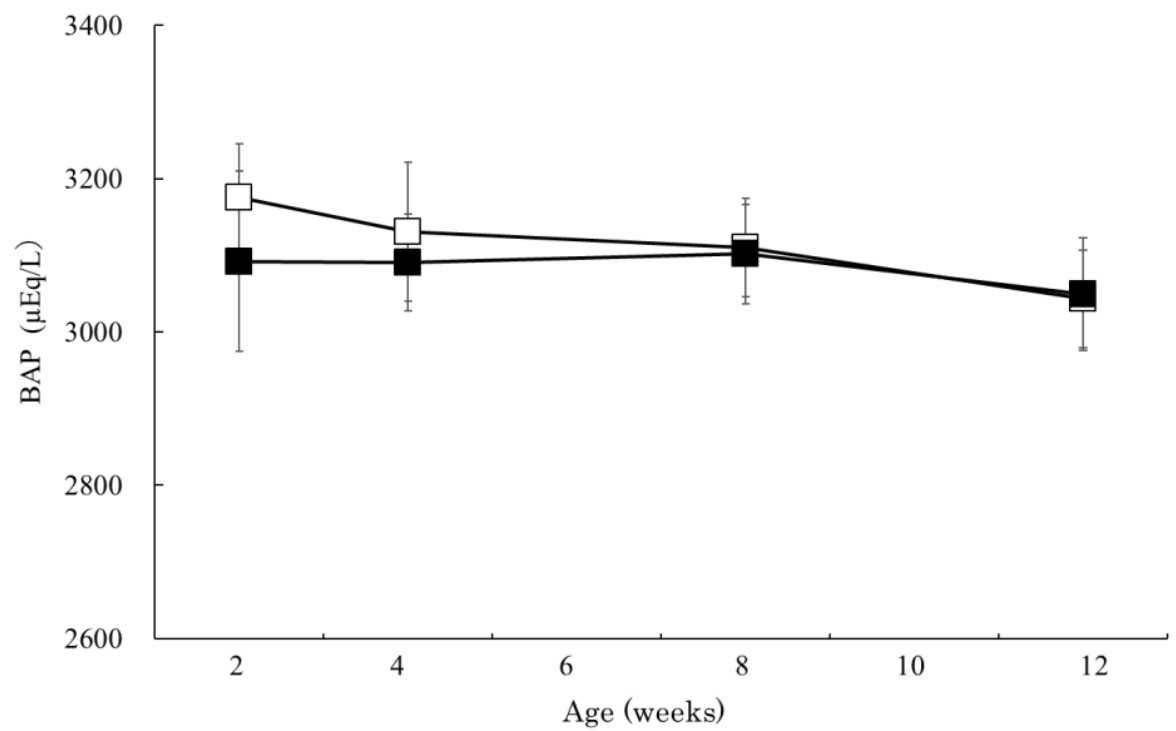

Fig. 2. Changes in serum biological antioxidant potential (BAP) in the VC group (solid squares) and the control group (hollow squares)

Data are shown as mean \pm SE 


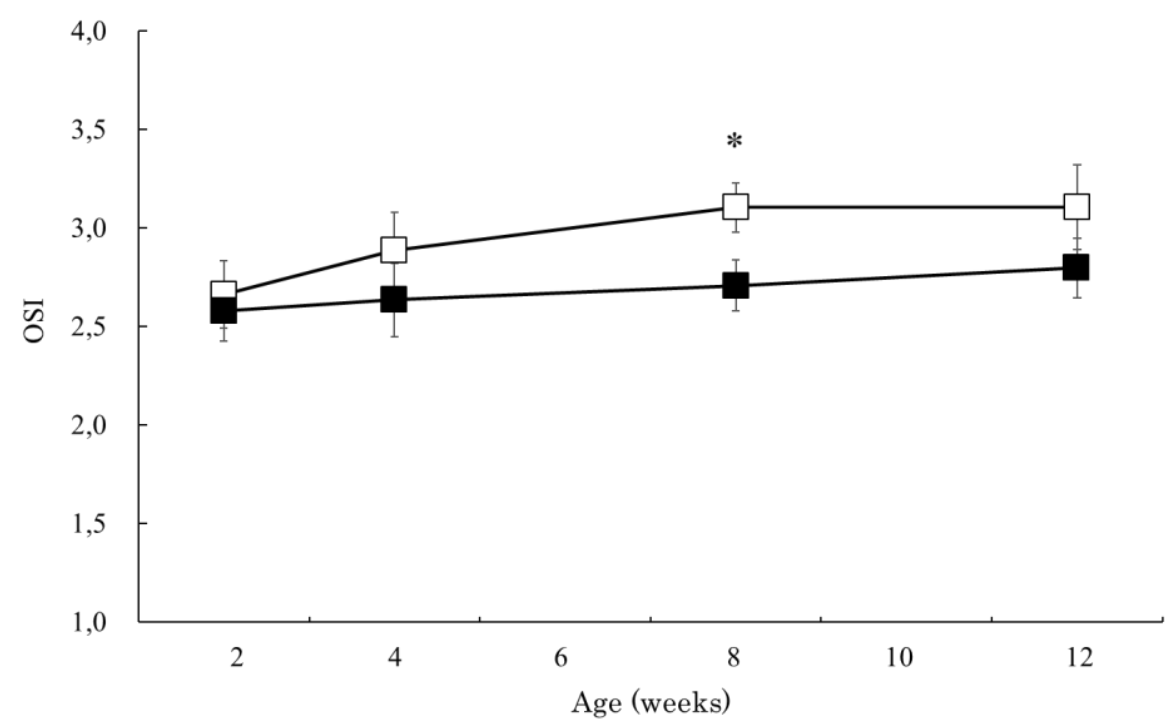

Fig. 3. Changes in oxidative stress index (OSI) in the VC group (solid squares) and the control group (hollow squares)

The asterisk indicates a significant difference between groups at the same age $(\mathrm{P}<0.05)$

Data are shown as mean $\pm \mathrm{SE}$

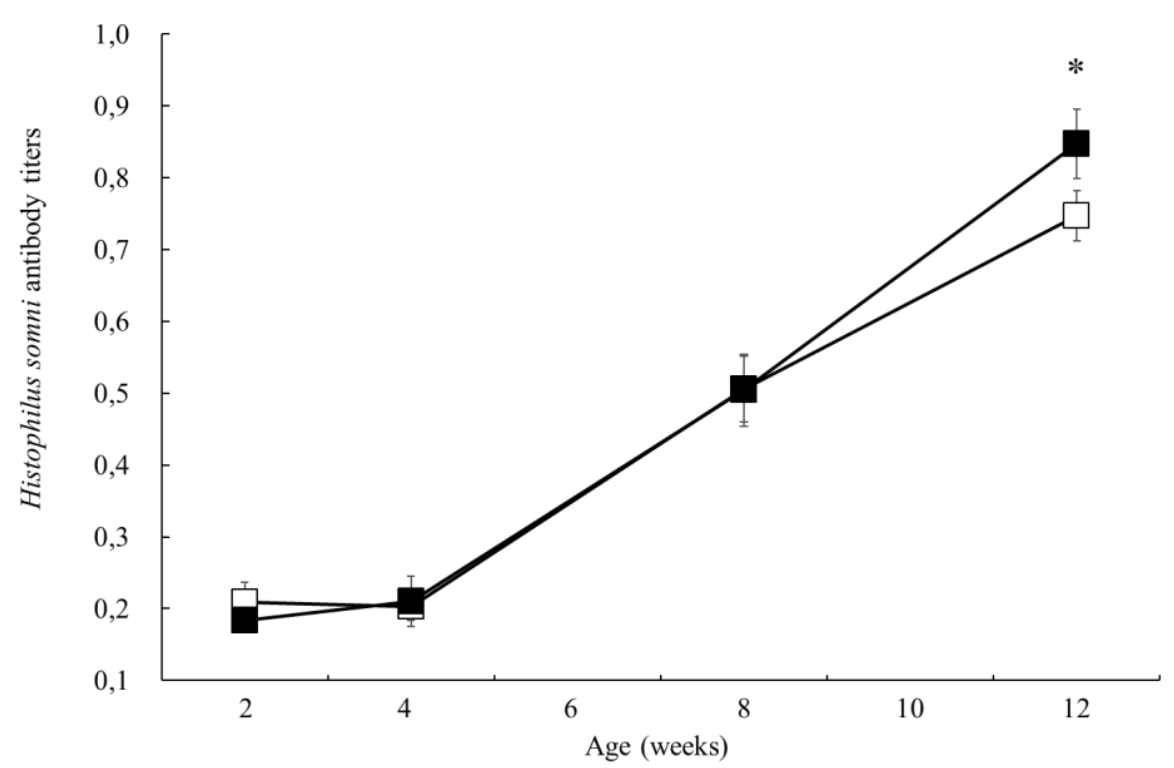

Fig. 4. Changes in antibody titres against Histophilus somni in the VC group (solid squares) and the control group (hollow squares)

The asterisk indicates a significant difference between groups at the same age $(\mathrm{P}<0.05)$

Data are shown as mean \pm SE

\section{Discussion}

In this study, the values of $\mathrm{WBC}, \mathrm{RBC}, \mathrm{Hb}$ and $\mathrm{Ht}$ in calves of both groups were within the normal range and there was no significant difference between the groups. All calves were also clinically healthy during the experiment period. Therefore, it is considered that this study was conducted without negatively affected by vitamin $\mathrm{C}$ supplementation to calves.

Oxidative stress is indicated by the concentration balance of reactive oxygen species (ROS) and antioxidants (12). It develops when production of reactive oxygen species increases and amounts of antioxidants decrease (12). The d-ROMs and BAP can easily be measured, and tests for them can be applied to gain an understanding of the clinical status of oxidative stress $(1,18,21)$. High d-ROMs values indicate increased production of ROS, and high BAP values indicate increased antioxidant capacity. Because these parameters are interdependent (12), it is also important to evaluate ROS and antioxidants jointly rather than separately, and from the d-ROMs and BAP values the grade of oxidative stress can be evaluated using the oxidative stress index (OSI) $(1,21)$. Vitamin C plays an important role as an antioxidant (15), efficiently contributes to defence against lipid peroxidation, and 
scavenges peroxyl radicals $(4,15,22)$. A previous study in humans has also shown that supplementation with vitamin $\mathrm{C}$ decreased several oxidative stress marker concentrations in blood (24). In this study, the d-ROMs and OSI at 8 weeks of age were significantly lower in the VC group than those in the control group. Therefore, vitamin $\mathrm{C}$ supplementation in the $\mathrm{VC}$ group might have contributed to oxidative stress reduction.

Several studies revealed that vitamin $\mathrm{C}$ has immunomodulatory functions $(4,8,19,22,23)$. Vitamin $\mathrm{C}$ supplementation to animals enhanced phagocytosis of macrophages and proliferated helper T cells and B cells $(4,8)$. Ascorbic acid also activates dendritic cells which present antigens to helper T cells in an antigen-antibody reaction and those cells are responsible for antibody production (10). Clinical trials in mice and humans revealed that vitamin $\mathrm{C}$ supplementation was associated with a significant increase in serum $\operatorname{IgG}$ concentration (20, 23). Furthermore, guinea pigs which received vitamin $\mathrm{C}$ supplementation produced more antibodies to the inoculum (19). In general, the inactivated vaccine is administered at least twice in order to obtain a booster effect (5). In this study, administration of the inactivated vaccine to calves was performed twice, at 4 and 8 weeks of age. As a result, at 8 weeks of age before the second vaccination there was no difference in antibody titres between the two groups, but at 12 weeks of age after the second vaccination, the VC group had a significantly higher antibody titre than the control group. Therefore, vitamin C supplementation to Japanese Black calves may enhance the booster effect against vaccination. It has also been reported that in Brown Swiss calves, antibody production was promoted by administration of vitamin $\mathrm{C}$ in addition to vitamins $\mathrm{A}, \mathrm{D} 3$ and $\mathrm{E}$ at the time of foot-and-mouth disease vaccination (11).

In conclusion, vitamin $\mathrm{C}$ supplementation to calves may reduce the blood cell oxidative stress and enhance antibody production after the second vaccination with inactivated $H$. somni. However, further studies are needed to clarify how it affects the blood oxidative metabolism and immune functions in the calves.

Conflict of Interests Statement: The authors declare that there is no conflict of interests regarding the publication of this article.

Financial Disclosure Statement: This study was supported by grants from a project of the National Agricultural Research Organization, Bio-oriented Technology Research Advancement Institution, Japan.

Animal Rights Statement: The present study was carried out in accordance with Kagoshima University animal care guidelines.

Acknowledgements: We would like to acknowledge Dr. Takahiro Kaneshige of Kyoto Biken Laboratories for his generous help.

\section{References}

1. Abuelo A., Hernández J., Benedito J.L., Castillo C.: Oxidative stress index (OSi) as a new tool to assess redox status in dairy cattle during the transition period. Animal 2013, 7, 1374-1378, doi: $10.1017 /$ S1751731113000396.

2. Allen J. W., Viel L., Bateman K.G., Rosendal S., Shewen P.E., Physick-Sheard P.: The microbial flora of the respiratory tract in feedlot calves: associations between nasopharyngeal and bronchoalveolar lavage cultures. Can J Vet Res 1991, 55, 341-346.

3. Canto J., Biberstein E.L., Schulte T.A., Behymer D.: Crossreactivity of Haemophilus somnus antibody in agglutination and complement fixation tests and in the enzyme-linked immunosorbent assay. J Clin Microbiol 1983, 17, 500-506.

4. Carr A.C., Maggini S.: Vitamin C and immune function. Nutrients 2017, 9, 1211, doi: 10.3390/nu9111211.

5. Chase C.C., Hurley D.J., Reber A.J.: Neonatal immune development in the calf and its impact on vaccine response. Vet Clin North Am Food Anim Pract 2008, 24, 87-104, doi: 10.1016/j.cvfa.2007.11.001.

6. Corbeil L.B., Widders P.R., Gogolewski R., Arthur J., Inzana T.J., Ward A.C.: Haemophilus somnus: bovine reproductive and respiratory disease. Can Vet J 1986, 27, 90-93.

7. Hemingway D.C.: Vitamin $\mathrm{C}$ in the prevention of neonatal calf diarrhea. Can Vet J 1991, 32, 184.

8. Hesta M., Ottermans C., Krammer-Lukas S., Zentek J., Hellweg P., Buyse J., Janssens G.P.: The effect of vitamin C supplementation in healthy dogs on antioxidative capacity and immune parameters. J Anim Physiol Anim Nutr 2009, 93, 26-34, doi: 10.1111/j.14390396.2007.00774.x.

9. Kampen A.H., Olsen I., Tollersrud T., Storset A.K., Lund A.: Lymphocyte subpopulations and neutrophil function in calves during the first 6 months of life. Vet Immunol Immunopathol 2006, 113, 53-63, doi: 10.1016/j.vetimm.2006.04.001.

10. Kim H.W., Cho S.I., Bae S., Kim H., Kim Y., Hwang Y.I., Kang J.S., Lee W.J.: Vitamin C up-regulates expression of CD80, CD86 and MHC Class II on dendritic cell line, DC-1 via the activation of p38 MAPK. Immune Netw 2012, 12, 277-283, doi: 10.4110/in.2012.12.6.277.

11. Kizil O., Gul Y.: Effects of vitamin AD3E and C on immune responses of cattle to trivalent foot and mouth disease vaccine. Revue Med Vet 2008, 159, 599-602.

12. Li R., Jia Z., Trush M.A.: Defining ROS in Biology and Medicine. React Oxyg Species 2016, 1, 9-21, doi: 10.20455/ros.2016.803.

13. Ministry of Agriculture, Factory and Fisheries Research Council Secretariat: Japanese feeding standard for beef cattle (in Japanese). Japan Livestock Industry Association, Tokyo, 2008.

14. Mori K., Kato T., Yokota O., Ohtsuka H.: Field trial of primary and booster dose of inactivated vaccine against bovine respiratory bacteria in young Holstein calves. J Vet Res 2020, 64, 223-230, doi: 10.2478/jvetres-2020-0042.

15. Niki E.: Action of ascorbic acid as a scavenger of active and stable oxygen radicals. Am J Clin Nutr 1991, 54, 1119S-1124S, doi: 10.1093/ajcn/54.6.1119s.

16. Nonnecke B.J., Waters W.R., Foote M.R., Palmer M.V., Miller B.L., Johnson T.E., Perry H.B. Fowler M.A.: Development of an adult-like cell-mediated immune response in calves after early vaccination with Mycobacterium bovis bacillus Calmette-Guérin. J Dairy Sci 2005, 88, 195-210, doi: 10.3168/jds.S00220302(05)72678-3.

17. Ohtsuka H., Ono M., Saruyama Y., Mukai M., Kohiruimaki M., Kawamura S.: Comparison of the peripheral blood leukocyte population between Japanese Black and Holstein calves. Anim Sci J 2011, 82, 93-98, doi: 10.1111/j.1740-0929.2010.00833.x.

18. Otomaru K., Ogawa R., Oishi S., Iwamoto Y., Hong H., Nagai K., Hyakutake K., Kubota C., Kaneshige T.: Effect of beta-carotene supplementation on the serum oxidative stress biomarker and antibody titer against live bovine respiratory syncytial virus 
vaccination in Japanese Black calves. Vet Sci 2018, 5, 102, doi: 10.3390/vetsci5040102.

19. Prinz W., Bloch J., Gilich G., Mitchell G.: A systematic study of the effect of vitamin $\mathrm{C}$ supplementation on the humoral immune response in ascorbate-dependent mammals. I. The antibody response to sheep red blood cells (a T-dependent antigen) in guinea pigs. Int J Vitam Nutr Res 1980, 50, 294-300.

20. Prinz W., Bortz R., Bregin B., Hersch M.: The effect of ascorbic acid supplementation on some parameters of the human immunological defense system. Int J Vitam Nutr Res 1977, 47, 248-257.

21. Ranade R., Talukder S., Muscatello G., Celi P.: Assessment of oxidative stress biomarkers in exhaled breath condensate and blood of dairy heifer calves from birth to weaning. Vet J 2014, 202, 583-587, doi: 10.1016/j.tvj1.2014.10.025.

22. Smirnoff N.: Ascorbic acid metabolism and functions: A comparison of plants and mammals. Free Radic Biol Med 2018, 122, 116-129, doi: 10.1016/j.freeradbiomed.2018.03.033.

23. Wu M., He M., Kang Y.: Vitamin C supplementation improved the efficacy of foot-and-mouth disease vaccine. Food Agric Immunol 2018, 29, 470-483, doi: 10.1080/09540105.2017. 1406459.

24. Yang C.C., Hsu S.P., Wu M.S., Hsu S.M., Chien C.T.: Effects of vitamin $\mathrm{C}$ infusion and vitamin E-coated membrane on hemodialysis-induced oxidative stress. Kidney Int 2006, 69, 706-714, doi:10.1038/sj.ki.5000109. 